\title{
On Robin boundary conditions and the Morse potential in quantum mechanics
}

\author{
B. Belchev, M.A. Walton \\ Department of Physics and Astronomy, University of Lethbridge \\ Lethbridge, Alberta, Canada T1K $3 M_{4}$ \\ borislav.belchev@uleth.ca,walton@uleth.ca
}

October 29, 2018

\begin{abstract}
The physical origin is investigated of Robin boundary conditions for wave functions at an infinite reflecting wall. We consider both Schrödinger and phase-space quantum mechanics (a.k.a. deformation quantization), for this simple example of a contact interaction. A non-relativistic particle moving freely on the half-line is treated as moving on the full line in the presence of an infinite potential wall, realized as a limit of a Morse potential. We show that the wave functions for the Morse states can become those for a free particle on the half-line with Robin boundary conditions. However, Dirichlet boundary conditions (standard walls) are obtained unless a mass-dependent fine tuning (to a reflection resonance) is imposed. This phenomenon was already observed for piece-wise flat potentials, so it is not removed by smoothing. We argue that it explains why standard quantum walls are standard. Next we consider the Wigner functions (the symbols of both diagonal and off-diagonal density operator elements) of phase-space quantum mechanics. Taking the (fine-tuned) limit, we show that our Wigner functions do reduce to the expected ones on the half-line. This confirms that the Wigner transform should indeed be unmodified for this contact interaction.
\end{abstract}

PACS: 03.65.-w, 03.65.Db, 03.65.Sq, 03.65.Nk 


\section{Introduction}

Point interactions and reflecting walls are known as contact interactions [1]. In quantum mechanics, they have been a subject of some interest lately-see [2] for some intriguing properties. Perhaps the simplest example is an infinite reflecting wall [1].

Contact interactions are described by potentials with sharp features. A smooth interaction can be encoded in a potential feature of a certain width $w$. A contact interaction is obtained for zero width $w$, or, equivalently, infinite sharpness $\alpha:=1 / w$. Physically, these sharp features should be understood in terms of the limit $w \rightarrow 0(\alpha \rightarrow \infty)$.

But can systems with sharp features be quantized after the limit is taken, or is it necessary to quantize before? Do we take $\alpha \rightarrow \infty$ before or after quantization:1]

If one takes the sharp limit $\alpha \rightarrow \infty$ before quantization, one can rely on mathematical conditions to proceed. In operator quantum mechanics, one only needs to impose appropriate boundary conditions on wave functions in coordinate space. The boundary conditions conserve probability and can be understood as necessary for self-adjointness of Hermitian operators, like the Hamiltonian, or extensions thereof 2 For the infinite reflecting wall, Robin boundary conditions are the only possibilities. They include the standard Dirichlet boundary conditions and the Neumann conditions as two extremal points in a one-parameter continuum of possibilities.

It has been emphasized that the non-standard versions of such interactions should not be ignored, since they may describe interesting physics [6, 1]. However, physical considerations such as symmetry (such as time-reversal invariance, e.g.) can eliminate possibilities in some cases [7]. Can the physical possibilities be restricted in other ways?

Our point of view is that physically, zero-width (or sharp) features must be understood fundamentally as $\alpha \rightarrow \infty$ limits of nonzero-width (smooth) ones. That is, the sharp case is an idealization, whose treatment should only provide a shortcut to the results obtained in the physical limit.

\footnotetext{
1 One way to anticipate that it does make a difference is to realize that the classical limit $\hbar \rightarrow 0$ and the sharp limit $\alpha \rightarrow \infty$ do not commute. The wave phenomenon of non-Newtonian scattering 3 4 makes that plain. For particle energy exceeding a discontinuous potential, there is a non-zero probability of reflection off the sharp feature, even though the process does not occur classically. Most strikingly, the probability is independent of Planck's constant, and so does not vanish as $\hbar \rightarrow 0$.

2 See [5] for nice expositions of the theory of self-adjoint extensions.
} 
In that spirit, the infinite potential wall was described by a limit of the Liouville potential in [8]. The standard wall, with Dirichlet boundary conditions, was recovered. In this work, we will extend the result of [8] to the general case of Robin boundary conditions, by generalizing the Liouville potential to a Morse potential.

In agreement with the results of others [9, 1, mass-dependent fine tuning is found to be necessary for non-standard walls to emerge. We believe that this fine tuning explains why standard quantum walls, with their Dirichlet boundary conditions, are standard. Non-standard walls are unlikely to be realized physically, because the required fine tuning is improbable 3

Our original motivation came from phase-space quantum mechanics (a.k.a. deformation quantization) 4 A further complication arises from sharp potential features in this context [13]5 We therefore also examine Wigner functions for non-standard and standard walls, using the Morse potential.

Dias and Prata [13] treated the special (standard) case of Dirichlet boundary conditions for the Schrödinger wave functions. To describe the complication they found, let $\rho(x, p)$ denote the Wigner function. At finite $\alpha$, it can be found two ways. First, one can start from the wave functions $\psi(x)$, and build the corresponding density operator. Then a Wigner transform will yield the Wigner function; denote the result $\rho_{\alpha}[\psi](x, p)$. Alternatively, one can use the dynamical equations of phase-space quantum mechanics. The $*$-eigenvalue equations can be solved, to yield $\rho_{\alpha}[*](x, p)$. As long as $\alpha<\infty$, we must have

$$
\rho_{\alpha}[\psi](x, p)=\rho_{\alpha}[*](x, p)
$$

For the case $\alpha=\infty$, Dias and Prata found

$$
\rho_{\infty}[\psi](x, p) \neq \rho_{\infty}[*](x, p)
$$

They then assumed that the Wigner transform $\rho_{\infty}[\psi](x, p)$ was unaltered and added a boundary potential so that the $*$-eigenvalue equations were compatible. That is, they modified the $*$-eigenvalue equations so that their solutions were $\tilde{\rho}_{\infty}[*](x, p)=\rho_{\infty}[\psi](x, p)$.

In an effort to justify their somewhat ad hoc approach, alternatives to the *-eigenvalue equations were found in [14. Dias and Prata then demonstrated

\footnotetext{
3 This is reminiscent of the result of [10], where the renormalization of a different singular interaction was shown to select a preferred self-adjoint extension.

4 See [1] for a review and [12] for a pedagogical introduction, e.g.

5 Perhaps this is not surprising, since even the corresponding classical trajectories are continuous in configuration space but discontinuous in phase-space.
} 
[15] that the use of the alternative, so-called $*$-eigen- $*$ value equations, had a certain equivalence to their treatment. Since the $*$-eigen- $*$ value equations were derived, rather than postulated, those arguments provided an indirect justification of their procedure.

More directly, the limit of the Liouville potential was studied in [8]. It was shown there that the Wigner transform of the wave functions with Dirichlet boundary conditions was indeed physical, as was assumed by Dias and Prata [13. That is,

$$
\lim _{\alpha \rightarrow \infty} \rho_{\alpha}[\psi](x, p)=\lim _{\alpha \rightarrow \infty} \rho_{\alpha}[*](x, p)=\rho_{\infty}[\psi](x, p) .
$$

The first equality was guaranteed, by (1), but the second was not. If the limit had produced $\rho_{\infty}[*](x, p)$ instead, for example, then the Wigner transform would have had to be modified, rather than the $*$-eigenvalue equations.

In this work, we will extend the result (3) of 8 to the general case of Robin boundary conditions.

Let us also mention that in [8], the connection was first made between selfadjoint extensions and the problem (2) found by Dias and Prata [13. Subsequently, those authors were able to show that the Hamiltonians that included the boundary potentials they introduced were indeed self-adjoint [16].

This paper is organized as follows. In the next section, we review the realization of Robin boundary conditions in limits of piece-wise flat potentials, following [9, 1]. There, mass-dependent fine tuning of the potential was found to be necessary to realize a non-standard wall, i.e. to avoid the standard Dirichlet boundary conditions. We point out that this fine tuning is equivalent to selecting a reflection resonance, as defined in [17.

In section 3, the analogous calculation is carried out for a smooth Morse potential. The Robin boundary conditions are recovered, with the same kind of mass-dependent fine tuning already found in $[9,1$. We also show that reflection resonances are again selected in the smooth case.

In section 4, Wigner functions for the Morse potential are considered. Using our solutions of the $*$-eigenvalue equations, described in [18, we show that the Wigner functions reduce to the expected ones [4] in the appropriate limit. That is, eqn. (3) is indeed obeyed.

The final section is our conclusion. 


\section{Robin boundary conditions from a discontinuous potential}

Consider a non-relativistic quantum particle that is confined to the positive half-line with coordinate $x$, but is otherwise free. Its wave function must satisfy the so-called Robin boundary conditions

$$
\psi(0)+L \psi^{\prime}(0)=0
$$

for some real length parameter $L \in(-\infty, \infty) \cup\{\infty\}$. The Robin, or mixed boundary conditions generalise the Dirichlet $(L=0)$ and Neumann $(L \rightarrow \pm \infty)$ ones. They conserve probability and realize the self-adjoint extension of the Hermitian Hamiltonian $H=p^{2} / 2 m$ on the half-line.

Though there is no mathematical reason other than simplicity to prefer them, Dirichlet boundary conditions are the most commonly applied. For that reason, infinite walls with other boundary conditions imposed are known as non-standard walls [1]. In this paper we investigate the physical motivation for standard and non-standard walls.

The real wave function

$$
\psi_{k}(x)=\sin (k x+\phi)
$$

obeys the boundary condition (4) if the phase is chosen so that

$$
k L=-\tan \phi .
$$

It is appropriate for an unbound particle of energy $\hbar^{2} k^{2} / 2 m$. For the same dynamics, one bound state also exists, with (unnormalized) wave function $e^{-x / L}$ and energy $-\hbar^{2} / 2 m L^{2}$, provided $L>0$.

The bound state provides the length scale $L$ : its energy defines it, and its wave function has range $L$. This does not work for $L<0$, however. A more democratic interpretation is provided by the Wigner time delay (advance)

$$
\delta t=2 \hbar \frac{d \phi}{d E}=-\frac{2 m L}{\hbar k\left(1+k^{2} L^{2}\right)},
$$

for $L>0(L<0) 6$

Let us now consider a particle moving on the (whole) real line with coordinate $x$ and Hamiltonian

$$
H=p^{2} / 2 m+V(x) .
$$

\footnotetext{
${ }^{6}$ See [1] and references therein.
} 
A particle with energy $2 m E=\hbar^{2} k^{2}$ has a time-independent wave function $\psi(x)$ satisfying the stationary Schrödinger equation

$$
-\frac{\hbar^{2}}{2 m} \frac{d^{2} \psi(x)}{d x^{2}}+V(x) \psi(x)=\frac{\hbar^{2} k^{2}}{2 m} \psi(x) .
$$

We will show that Robin boundary conditions can arise from the limit of a smooth potential. This generalizes the derivation of Dirichlet boundary conditions from the sharp $\alpha \rightarrow \infty$ limit of the Liouville potential $V_{\alpha}(x)=\frac{\hbar^{2} \kappa^{2}}{2 m} e^{-2 \alpha x}$. In the context of deformation quantization, the latter result was obtained in [8].

To prepare for that calculation, we'll first study a discontinuous, piece-wise flat potential:

$$
V_{\alpha}(x)=\left\{\begin{array}{cc}
\infty, & x<0 \\
-\frac{\hbar^{2} \kappa^{2}}{2 m} \alpha \ell(\alpha \ell+1), & 0 \leq x \leq 1 / \alpha, \\
0, & x>1 / \alpha .
\end{array}\right.
$$

Here $\ell, 1 / \alpha$ and $1 / \kappa$ are lengths, with $\kappa^{2}>0$ controlling the overall strength of the potential. Seba 9 showed that when this potential becomes an infinite wall as $\alpha \rightarrow \infty$, Robin boundary conditions are recovered.

To see this, solve the Schrödinger equation piece-wise to get

$$
\psi_{\alpha}(x)=\left\{\begin{array}{cc}
0, & x<0 \\
\sin \left(x \sqrt{k^{2}+\kappa^{2} \alpha \ell(\alpha \ell+1)}\right), & 0 \leq x \leq 1 / \alpha, \\
A \sin (k x+\phi), & x>1 / \alpha
\end{array}\right.
$$

for an energy $E=\hbar^{2} k^{2} / 2 m>0$. Notice that the boundary conditions at $x=0$ are Dirichlet. Those at $x=1 / \alpha$, however, are of the mixed type, i.e., Robin. We can therefore derive Robin boundary conditions at $x=0_{+}:=\lim _{\alpha \rightarrow \infty} 1 / \alpha$. From the point of view of the physical wave function outside the resulting point interaction, it is the Robin (instead of the Dirichlet) boundary conditions that must be imposed.

Matching the wave-function values and derivatives at $x=1 / \alpha$, and taking the large $\alpha$ limit gives

$$
\kappa=\kappa_{n}:=\frac{\pi}{\ell}\left(n+\frac{1}{2}\right), n \in \mathbb{Z} .
$$

Then $\sin \left(\kappa_{n} \ell\right)=(-1)^{n}$, and we find

$$
A=A_{n}:=\sqrt{1+\frac{\pi^{4}\left(n+\frac{1}{2}\right)^{4}}{4 k^{2} \ell^{2}}}, \quad \tan \phi=\tan \phi_{n}=-\frac{2 k \ell}{\pi^{2}\left(n+\frac{1}{2}\right)^{2}} .
$$

Comparing to (6), we get

$$
L=L_{n}:=\frac{2 \ell}{\pi^{2}\left(n+\frac{1}{2}\right)^{2}}
$$


for the Robin length scale.

So the Robin boundary conditions are found for $x=0_{+}$, but only barely: there are solutions only for a discrete set of values of $\kappa$, indexed by the integer $n$. The strength of the potential needs to be finely tuned, tuned differently for different particle masses 7 and the non-standard Robin boundary conditions arise for a very limited subset of possible parameters.

What is the physical significance of the fine tuning? It selects a resonance. For this potential the probability of reflection is always one, but a reflection resonance can still be defined by a rapid change of $\pi$ in the phase shift [17]. From the matching conditions we can derive

$$
\frac{\tan (j / \alpha)}{j / \alpha}=\frac{\tan (k / \alpha+\phi)}{k / \alpha}
$$

where $j:=\left[k^{2}+\kappa^{2} \alpha \ell(\alpha \ell+1)\right]^{1 / 2}$. Demanding that $0=\frac{d^{2} \phi}{d \phi^{2}}$, and selecting the maxima of $\frac{d \phi}{d \phi}$, leads to $\tan (j / \alpha)=\infty$, or

$$
\frac{j}{\alpha}=\frac{\left[k^{2}+\kappa^{2} \alpha \ell(\alpha \ell+1)\right]^{1 / 2}}{\alpha}=\left(n+\frac{1}{2}\right) \pi, \quad n \in \mathbb{Z} .
$$

In the $\alpha \rightarrow \infty$ limit, the fine-tuning condition (12) is recovered.

Let us note that the reflection resonance condition (16) corresponds to Neumann boundary conditions at $x=1 / \alpha$, even before the $\alpha \rightarrow \infty$ limit is taken. Of course, the requirement (12) for Robin boundary conditions does not select Neumann boundary conditions. Substituting (12) yields

$$
\frac{j}{\alpha}=\left(n+\frac{1}{2}\right) \pi+\left(\alpha L_{n}\right)^{-1}+\mathcal{O}\left(\alpha^{-2}\right),
$$

using (14). This shows that the fine tuning is to near a reflection resonance; how it is approached in the $\alpha \rightarrow \infty$ limit determines the Robin length scale $L_{n}$ and so the boundary condition that is realized.

Let us also consider the bound states of the Šeba potential in the $\alpha \rightarrow \infty$ limit. For the negative energy states the wave function will decay exponentially in the interval $x \in(1 / \alpha, \infty)$. Dividing the matching conditions for the wave function and its derivative yields

$$
\begin{gathered}
\sqrt{-2 m|E| / \hbar^{2}+\kappa^{2} \alpha \ell(\alpha \ell+1)} \cot \left(\frac{1}{\alpha} \sqrt{-2 m|E| / \hbar^{2}+\kappa^{2} \alpha \ell(\alpha \ell+1)}\right) \\
=-\sqrt{2 m|E| / \hbar^{2}} .
\end{gathered}
$$

7 For the standard wall with Dirichlet boundary conditions, this mass dependence is not present. 
As before we can compare the coefficients in front of the different powers of $\alpha$. The only possible energy is then $E=-\hbar^{2} \kappa^{4} \ell^{2} / 8 m$. Taking into account that (12 14) are needed for the Robin boundary conditions to arise, we obtain the correct energy $-\hbar^{2} / 2 m L^{2}$ in the $\alpha \rightarrow \infty$ limit. The bound state energy is recovered from the Šba potential, for $L>0$. We can also verify that, for the same values of the parameters, the (unnormalized) wave function of the unique bound state is $e^{-x / L}$ in the limit.

One criticism of these results could be that Dirichlet boundary conditions were assumed, not derived for the infinite wall (with no extra structure) at $x=0$ in the Šeba potential. In addition, infinite potential walls are only idealisations of very high, but finite walls, and so the infinite wall should be treated as the limit of a finite wall. However, similar results were obtained later in [1] but with a finite wall, and no particular boundary conditions assumed. Robin boundary conditions were again obtained, with non-standard walls arising only when a mass-dependent fine tuning was imposed. We will therefore study here a nonsharp, or smoothed version of the Šeba potential, rather than of the potential in [1].

The authors of [1] speculate that a better choice than their piece-wise flat, discontinuous potential might eliminate the peculiar mass-dependent fine tuning required for non-standard walls. Presumably, it could also be argued to be possible for the Šeba potential [9]. We will find, however, that the mass-dependent fine tuning remains necessary in a smoothed version of Šeba's potential. In retrospect, this should perhaps not be surprising, at least for Schrödinger quantum mechanics. The limit that squeezes and stretches the potentials into an infinite wall is so extreme, it seems unimportant whether the original potential has corners or is smoothed.

\section{Wave functions with Robin boundary conditions from a Morse potential}

To study how Robin boundary conditions arise as limits in deformation quantization, sharp potential features should be avoided. We will now carry out an analysis similar to that of the previous section, but for a smooth potential. The spectrum is first found for a potential with undetermined parameters. Then

we consider a certain limit of the parameters, demanding that we recover the infinite wall, and that the states realized coincide with the eigenstates for the infinite wall, with Robin boundary conditions obeyed. 
With its short range repulsion and longer range attraction, the smooth Morse potential

$$
V(x)=\frac{\hbar^{2} \kappa^{2}}{2 m}\left(e^{-2 \alpha x}-b e^{-\alpha x}\right)
$$

is a rough approximation to Šeba's. Besides $\alpha$, two more parameters are needed $-\kappa$ determines the overall potential strength, $b \geq 0$ the position of the well, and together they fix its depth. We will need to impose conditions on these coefficients in order to obtain Robin boundary conditions in the large $\alpha$ limit.

The previous section indicates that we need only show that Robin boundary conditions apply at $x=\epsilon$, where epsilon is very small, but beyond the features of the Morse potential when $\alpha \rightarrow \infty$. For the unbound wave functions, therefore, we need only require that the relevant wave functions have the asymptotic form $\psi(x) \sim A \sin (k x+\phi)$ as $x \rightarrow \infty$, with $\phi$ variable.

To do that we first need to find the unbound wave functions. Following Matsumoto [19], we can solve the stationary Schrödinger equation for the Morse potential (19). The substitution $\psi(x)=\phi(z), z=\exp (-\alpha x)$, changes the Schrödinger equation into

$$
z^{2} \phi^{\prime \prime}+z \phi^{\prime}+\frac{1}{\alpha^{2}}\left[\frac{2 m E}{\hbar^{2}}-\kappa^{2} z^{2}+\kappa^{2} b z\right] \phi=0 .
$$

This can be further transformed into canonical form (without a first derivative term) using the substitution $\phi(z)=z^{-1 / 2} F(z)$. Changing the variables to $y:=2 \kappa z / \alpha$ leads to the so-called Whittaker equation, treated in [20, Chapter XVI:

$$
f^{\prime \prime}+\left\{-\frac{1}{4}+\frac{b \kappa}{2 \alpha} \frac{1}{y}+\frac{1}{y^{2}}\left[\frac{1}{4}-\left(\frac{i k}{\alpha}\right)^{2}\right]\right\} f=0,
$$

where $f(y):=F(\alpha y / 2 \kappa)$ and, as before, $k=\sqrt{2 m E} / \hbar$. The two linearly independent solutions are defined in [21, p.755. They are called Whittaker functions and can be expressed in terms of the Tricomi confluent hypergeometric function $U(\mu, \nu, z)$ and the Kummer confluent hypergeometric function $M(\mu, \nu, z): 8$

$$
\begin{aligned}
M_{l m}(z) & =z^{m+1 / 2} e^{-z / 2} M(1 / 2+m-l, 1+2 m ; z), \\
W_{l m}(z) & =z^{m+1 / 2} e^{-z / 2} U(1 / 2+m-l, 1+2 m ; z) .
\end{aligned}
$$

8 The Whittaker function $M_{l m}(z)$ should not be confused with the Kummer function $M(\mu, \nu, z)$ in the above equation. Subscripts are used to denote the parameters of the Whittaker functions in the literature, and the explicit bracket notation is used for confluent hypergeometric functions. For further information involving the hypergeometric functions see [21, p.753 and 22, p.503-506. 
For our purposes, we only need the definitions of those functions

$$
\begin{gathered}
M(\mu, \nu ; z)=\sum_{n=0}^{\infty} \frac{(\mu)_{n}}{(\nu)_{n}} \frac{y^{n}}{n !} \\
U(\mu, \nu ; z)=\frac{\Gamma(\nu-1)}{\Gamma(\mu)} z^{1-\nu} M(1+\mu-\nu, 2-\nu ; z) \\
+\frac{\Gamma(1-\nu)}{\Gamma(\mu-\nu+1)} M(\mu, \nu ; z) .
\end{gathered}
$$

Here we use the Pochhammer symbol $(\mu)_{n}:=\mu(\mu+1) \ldots(\mu+n-1),(\mu)_{0}:=1$.

Now the wave function can be written as

$$
\psi_{k}(x)=e^{\alpha x / 2}\left[C_{1} M_{\frac{b \kappa}{2 \alpha}, \frac{i k}{\alpha}}(y(x))+C_{2} W_{\frac{b \kappa}{2 \alpha}, \frac{i k}{\alpha}}(y(x))\right] .
$$

Imposing reality yields $C_{1}=0$. The second term has physical asymptotic behaviour: for large positive $x$ it is sinusoidal with a phase depending on the potential parameters; for negative $x$ far from the origin, there is the expected rapid exponential decay of a classically forbidden region. The wave function is therefore

$$
\psi_{k}(x)=C e^{\alpha x / 2} W_{\frac{b \kappa}{2 \alpha}, \frac{i k}{\alpha}}\left(\frac{2 \kappa}{\alpha} e^{-\alpha x}\right) .
$$

With the help of equation (25) we can rewrite this result in a form similar to that given by Matsumoto in [19] for a Morse potential with $b=2$. The wave function is manifestly real in this form:

$$
\begin{aligned}
\psi(y) & =C e^{-y / 2} \tilde{A} y^{i k / \alpha} M\left(\frac{1}{2}-\frac{b \kappa}{2 \alpha}+\frac{i k}{\alpha}, 1+\frac{2 i k}{\alpha} ; y\right)+ \\
+ & C e^{-y / 2} \tilde{A}^{*} y^{-i k / \alpha} M\left(\frac{1}{2}-\frac{b \kappa}{2 \alpha}-\frac{i k}{\alpha}, 1-\frac{2 i k}{\alpha} ; y\right),
\end{aligned}
$$

with $C$ a real normalization constant, and

$$
\tilde{A}=\frac{\Gamma\left(-\frac{2 i k}{\alpha}\right)}{\Gamma\left(\frac{1}{2}-\frac{b \kappa}{2 \alpha}-\frac{i k}{\alpha}\right)} .
$$

Let us now examine the asymptotic behaviour of the wave function and how it depends on the parameters. In the limit $\alpha \rightarrow \infty, \exp (-y / 2) \sim \exp \left(-e^{-\alpha x}\right)$ approaches the step function, so the dynamics will be restricted to the positive half-line. The limit $x \rightarrow \infty$ corresponds to $y \rightarrow 0$. Using (28) and (24) we obtain

$$
\psi(x) \sim C|\tilde{A}| \cos [k x-\arg (\tilde{A})] .
$$


The phase can be calculated from (29) and Euler's infinite product formula

$$
\frac{1}{\Gamma(u)}=u e^{\gamma u} \prod_{n=1}^{\infty}\left[\left(1+\frac{u}{n}\right) e^{-u / n}\right] .
$$

A short calculation shows that

$$
\begin{aligned}
& \arg (\tilde{A})=\frac{\pi}{2}+\frac{\gamma k}{\alpha}- \\
& \sum_{n=0}^{\infty}\left\{\frac{k}{\alpha(n+1)}-\tan ^{-1}\left[\frac{2 k}{\alpha(n+1)}\right]+\tan ^{-1}\left[\frac{2 k}{(2 n+1) \alpha-b \kappa}\right]\right\} .
\end{aligned}
$$

Apart from the $\pi / 2$, all terms will vanish in the $\alpha \rightarrow \infty$ limit, except those of the form $\tan ^{-1}[2 k /((2 n+1) \alpha-b \kappa)]$. For one such term to survive the limit, we need $\kappa=\mathcal{O}\left(\alpha^{1}\right)$. If the strength $\kappa$ does not have this form, we will recover Dirichlet boundary conditions, i.e. the standard wall. Now, since it is $b \kappa$ that is relevant, we let $b$ absorb the proportionality constant, and use $\kappa=\alpha+\mathcal{O}\left(\alpha^{0}\right)$. Finally, because the terms of order $\alpha^{0}$ and lower will not affect the results, we drop them, and put $\kappa=\alpha$ from now on.

In order to realize Robin boundary conditions (4), the parameter $b$ must be of the special form

$$
b=(2 n+1)-2 L^{-1} / \alpha+\mathcal{O}\left(\alpha^{-2}\right) .
$$

Here $L$ is a fixed length, independent of $\alpha$. Then we find

$$
k L=\tan \arg (\tilde{A})
$$

in the large $\alpha$ limit, so that the wave function (28) does indeed satisfy the Robin boundary conditions (4).

At large $\alpha$ the term $2 L^{-1} / \alpha$ is negligible compared to the other two. While the parameter $b$ approaches an odd integer the second infinitesimal term is crucial. Apparently, we need to fine-tune the parameter $b$ to recover the Robin boundary conditions. A version of this phenomenon has already been encountered in [1] where the parameters can only take very limited values. The authors argue that fine tuning may be a result of the particular choice of potential they are using, possibly because it is not smooth. Since our analysis, using a smooth potential, produces a version of fine tuning as well, fine tuning cannot be related to discontinuity alone.

For non-standard walls, we must fine-tune the parameters so that we are near Neumann boundary conditions. Notice that this is precisely as it was for the Šeba potential of sect. 2 (see (17) and nearby). Put another way, the fine-tuning is again to a reflection resonance, or slightly off its peak. 
Let us now consider the bound states. Their wave functions are given in [23]

as

$$
\psi(x) \propto \exp \left(-\kappa e^{-\alpha x} / \alpha\right) e^{-\alpha(\nu-b \kappa / 2 \alpha+1 / 2) x} L_{\nu}^{b \kappa / \alpha-2 \nu-1}\left(2 \kappa e^{-\alpha x} / \alpha\right)
$$

where

$$
L_{n}^{\lambda}(x)=\sum_{m=0}^{n}(-1)^{m}\left(\begin{array}{c}
n+\lambda \\
n-m
\end{array}\right) \frac{x^{m}}{m !}
$$

are the associated Laguerre polynomials $L_{n}^{\lambda}(x)$. The energies are

$$
E_{\nu}=-\frac{\hbar^{2} \alpha^{2}}{2 m}(\nu-b \kappa / 2 \alpha+1 / 2)^{2},
$$

for integer $\nu \in[0,\lfloor b \kappa / 2 \alpha\rfloor]$, where $\lfloor a\rfloor$ is the smallest integer less than $a$.

Consider now the $\alpha \rightarrow \infty$ limit. The Laguerre polynomials are normalized to one at zero argument, and $\exp \left(-\kappa e^{-\alpha x} / \alpha\right)$ turns into the step function. The only term that remains to be analyzed is $e^{-\alpha(\nu-b \kappa / 2 \alpha+1 / 2) x}$. Clearly, $-\alpha(\nu-b \kappa / 2 \alpha+1 / 2)$ must be a negative constant (independent of $\alpha$ ) so that we have a normalizable wave function that does not disappear in the large $\alpha$ limit. Again we can set $\kappa=\alpha$, and the solving for $b$ yields precisely equation (33). Analyzing the bound states provides an alternative way of deriving the fine tuning condition.

On the other hand, let us give particular values to the constants in (33), i.e. fix $b$. All the bound states will vanish for $x>0$ except the one that has highest quantum number $\nu=\lfloor b / 2\rfloor$. This is because the maximal integer will cancel the integer part of $b$ and leave only the fine tuning part $-2(L \alpha)^{-1}$. The bound state wave function $\sim e^{-x / L}$ will be recovered with the correct energy $-\hbar^{2} / 2 m L^{2}$.

To summarize, in this section we demonstrated that the $\alpha \rightarrow \infty$ limit of the Morse potential (19) can be used to generate Robin boundary conditions. Fine tuning is necessary, however: the parameter $b$ must be an odd non-negative integer plus a term with $1 / \alpha$ asymptotics that determine the length scale $L$ of the Robin boundary condition. If the fine tuning is absent or if the integer part of $b$ is not an odd integer, then we can only recover Dirichlet boundary conditions, i.e. standard walls. A new observation is that the fine-tuning selects a reflection resonance.

Notice that the definition of $\kappa$ involves the particle mass. So, if the particle mass changes, so must the potential. The fine tuning required is also mass dependent. 
The situation is very similar to that for the discontinuous Šeba potential 9] treated in sect. 2, and to the results of [1]. The mass-dependent fine-tuning that is necessary for non-standard (Robin boundary condition) walls seems to be more than an artifact of the choice of potential. In particular, just smoothing out the discontinuities of a piece-wise flat potential is not sufficient to avoid this property. As already stated, this is perhaps reasonable in hindsight: it seems that the limit that squeezes and stretches the potentials into an infinite wall is so extreme that it is unimportant whether the original potential has corners or is smoothed.

\section{Wigner functions and Robin boundary conditions with a Morse potential}

In phase-space quantum mechanics (see [11, 12, e.g.), the Wigner function $\rho(x, p ; t)$ encodes all measurable information about the quantum state of a system. It satisfies the equation of motion

$$
i \hbar \partial_{t} \rho(x, p, t)=[H, \rho(x, p, t)]_{*},
$$

where $[H, \rho]_{*}=H * \rho-\rho * H$, and the Moyal $*$-product is defined by

$$
*=\exp \left\{\frac{i \hbar}{2}\left(\overleftarrow{\partial_{x}} \overrightarrow{\partial_{p}}-\overleftarrow{\partial_{p}} \overrightarrow{\partial_{x}}\right)\right\}
$$

It can be expressed as a linear combination of stationary Wigner functions with time-dependent coefficients:

$$
\rho(x, p, t)=\sum_{E_{L}, E_{R}} C_{E_{L} E_{R}} e^{-i\left(E_{L}-E_{R}\right) t / \hbar} \rho_{E_{L} E_{R}}(x, p) .
$$

Here $\rho_{E_{L} E_{R}}$ denotes the Hamiltonian $*$-eigenfunction that can be found by solving the system of equations:

$$
\begin{gathered}
H * \rho_{E_{L} E_{R}}(x, p)=E_{L} \rho_{E_{L} E_{R}}(x, p), \\
\rho_{E_{L} E_{R}}(x, p) * H=E_{R} \rho_{E_{L} E_{R}}(x, p) .
\end{gathered}
$$

Alternatively, the Wigner transform

$$
\rho_{E_{L} E_{R}}(x, p)=\int_{-\infty}^{\infty} d y e^{i y p}\left\langle x+\hbar y / 2 \mid E_{L}\right\rangle\left\langle E_{R} \mid x-\hbar y / 2\right\rangle
$$

allows them to be determined from the wave functions, if known. For smooth potentials, the resulting Wigner functions are known to agree. 
For discontinuous potentials, however, that is not necessarily the case [13. For the infinite wall (or a particle confined to the half-line), Dias and Prata [13] showed that the Wigner transform of the density operator only satisfies the $*$-eigenvalue equations if the free Hamiltonian is modified. No independent motivation was given for the change to the Hamiltonian, however. It was also assumed that the Wigner transform itself did not need to be adjusted.

An independent motivation was first suggested in [8]: self-adjointness of the Hamiltonian. The free Hamiltonian on the half-line is not self-adjoint. It does have self-adjoint extensions, however, and these correspond precisely to the possible boundary conditions (4) (see [5], e.g.). Subsequently, the selfadjointness of the Dias-Prata modified Hamiltonian was demonstrated in [16].

Here we are concerned with the assumption of an unmodified Wigner transform. That is, does the unmodified Wigner transform of the density operator provide the physical Wigner function? In [8], we answered in the affirmative, by treating the infinite wall as the limit of a smooth, Liouville potential. Only the standard Dirichlet boundary conditions were recovered, however. Here we will show that non-standard walls can be realized in a similar way, using the Morse potential, and that the naïve Wigner transform does indeed work, for all Robin boundary conditions, describing both non-standard and standard walls.

The Wigner transforms of the density operator elements relevant to Robin boundary conditions have already been computed, in [4. For $x>0$, using the wave functions (5, 6), we find:

$$
\begin{aligned}
\rho_{\infty}[\psi](x, p) \propto \frac{\sin [2(p / \hbar-k) x]}{(p / \hbar-k)}+\frac{\sin [2(p / \hbar+k) x]}{(} & \\
& +2 \cos \left(2 k x-\delta_{k}\right) \frac{\sin (2 x p / \hbar)}{p / \hbar} .
\end{aligned}
$$

In addition, the $*$-eigenvalue equations (41, 42) for the Morse Hamiltonian have also been solved in [18. We must take their limit $\alpha \rightarrow \infty$ as described in the last section, and compare with (44).

In [18, the $*$-eigenvalue equations (41, 42) were solved for the Morse potential using a Mellin transform and factorization 9 Writing

$$
E_{L}=: \frac{\hbar^{2} k_{L}^{2}}{2 m}, \quad E_{R}=: \frac{\hbar^{2} k_{R}^{2}}{2 m}
$$

the result was of the form

$\rho_{E_{L} E_{R}}(x, p) \propto$

\footnotetext{
9 The method used there should be useful for any potential that is a polynomial in an
} exponential. 


$$
\int_{c-i \infty}^{c+i \infty} d s\left(16 e^{4 \alpha x}\right)^{-s} w_{L}\left(s-\frac{i p}{2 \alpha \hbar}, k_{L}\right) w_{R}\left(s+\frac{i p}{2 \alpha \hbar}, k_{R}\right)
$$

The factors can be written using

$$
\begin{aligned}
& w_{I}\left(t, k_{I}\right) \propto \frac{4^{t+i k_{I} / 2 \alpha} \Gamma\left(-2 i k_{I} / \alpha\right)}{\Gamma\left(1 / 2-b / 2-i k_{I} / \alpha\right)} \Gamma\left(-2 t+i k_{I} / \alpha\right) \times \\
&{ }_{2} F_{1}\left(1 / 2-b / 2+i k_{I} / \alpha,-2 t+i k_{I} / \alpha ; 1+2 i k_{I} / \alpha ; 2\right)+ \\
& \frac{4^{t-i k_{I} / 2 \alpha} \Gamma\left(2 i k_{I} / \alpha\right)}{\Gamma\left(1 / 2-b / 2+i k_{I} / \alpha\right)} \Gamma\left(-2 t-i k_{I} / \alpha\right) \times \\
&{ }_{2} F_{1}\left(1 / 2-b / 2-i k_{I} / \alpha,-2 t-i k_{I} / \alpha ; 1-2 i k_{I} / \alpha ; 2\right),
\end{aligned}
$$

with $I=L, R$. Here we have defined $A=4^{i k_{I} / \alpha} \tilde{A}$, with $\tilde{A}$ as in (29), and used the identity $\Gamma(2 z) \propto 2^{2 z-1} \Gamma(z) \Gamma(z+1 / 2)$. This is the general solution of the *-eigenvalue equations of phase-space quantum mechanics, for unbound states in a Morse potential (19) with arbitrary real parameter $b$.

Following [8], we use the residue theorem to find the limit of the Wigner function when $\alpha \rightarrow \infty$. Since the calculation is straightforward but lengthy, we omit the details. The integrand of (46) has 4 terms, one proportional to $\tilde{A}^{2}$, one to $\tilde{A}^{* 2}$ and two to $|\tilde{A}|^{2}$. The $|\tilde{A}|^{2}$-terms yield contributions proportional to $\left[e^{2 i x(p / \hbar-k)}-e^{-2 i x(p / \hbar-k)}\right] /(p / \hbar-k)$ and $\left[e^{2 i x(p / \hbar+k)}-e^{-2 i x(p / \hbar+k)}\right] /(p / \hbar+k)$. The $\tilde{A}^{2}$-term and the $\tilde{A}^{* 2}$-term yield

$$
\hbar\left[e^{2 i \arg \tilde{A}+2 i x p / \hbar-2 i x k}-e^{2 i \arg \tilde{A}-2 i x p / \hbar-2 i x k}\right] / p
$$

and

$$
\hbar\left[e^{-2 i \arg \tilde{A}+2 i x p / \hbar+2 i x k}-e^{-2 i \arg \tilde{A}-2 i x p / \hbar+2 i x k}\right] / p
$$

Note that all the terms arising from residues at $i\left( \pm p / \hbar \pm^{\prime} k\right) / 2 \alpha+1 / 2$ produce decaying exponential factors and therefore do not contribute. Also, the Gauss hypergeometric function ${ }_{2} F_{1}(a, b ; c ; z)$ is analytic with respect to its second argument and $\tilde{\infty}$ is its only singularity. The contributions from ${ }_{2} F_{1}(a, b ; c ; z)$ will manifest themselves as a multiplication by constants in all cases. In particular, for those terms that survive in the limit of interest, the constant is 1 .

The algebra can now be completed to reproduce the Wigner function (44) for an infinite, but possibly non-standard wall as we hoped. The Robin boundary conditions are indeed recovered using the Morse potential. We have outlined how the calculation is done for diagonal elements of the symbol of the density operator, but the non-diagonal case works in similar fashion. 


\section{Conclusion}

Let us summarize our results.

In section 2, we reviewed Šeba's analysis [9] showing that Robin boundary conditions (for wave functions) could be realized by a limit $(\alpha \rightarrow \infty)$ of a discontinuous, piece-wise flat potential, eqn. (10). We pointed out that standard walls (Dirichlet boundary conditions) are generically realized in the sharp limit, and non-standard walls arise only if a mass-dependent fine-tuning (12) is imposed; these observations are in agreement with those made in 1, for the limit of a similar, but everywhere finite, potential 10 We observe that the parameters are fine-tuned to a reflection resonance in the limit. If the fine-tuning is imposed, then non-standard walls can be realized, and the Robin length scale $L$ is determined by exactly how the limit resonance is approached (see eqn. (17)).

The analysis of the piece-wise flat Šeba potential was repeated with a qualitatively similar, but smooth potential, the Morse potential of eqn. (19). Remarkably, the results were almost unchanged. Analysis of both the unbound and bound states yielded a mass-dependent fine tuning (33) required for nonstandard boundary conditions. Again, a reflection resonance is selected by the fine tuning, and how the resonance is approached in the $\alpha \rightarrow \infty$ limit determines the precise boundary conditions realized, i.e., the Robin length scale $L$.

As mentioned in the introduction, the infinite reflecting wall is perhaps the simplest example of a so-called contact interaction. For such, the interaction imposes boundary or matching conditions, such as the Robin boundary conditions on the half line. Alternatively, the same conditions can be found by demanding that the Hamiltonian or its extension be self-adjoint (see [5]).

Here we have assumed that contact interactions can only be realized physically as limits of smoother, less localized interactions 11 In agreement with [9, 1], mass-dependent fine tuning was found to be necessary for non-standard walls to emerge. We therefore believe this explains why standard quantum walls, with their Dirichlet boundary conditions, are standard. Non-standard walls are unlikely to be realized physically, because the required fine tuning is highly improbable.

It would be interesting to see if the realizations of other contact interactions as limits require similar fine tuning, and if there are other so-called standard boundary/matching conditions selected that way.

\footnotetext{
10 The independent derivation of the fine-tuning condition from a study of the bound states (see eqn. (18), instead of just the continuum, is perhaps new.

11 Clearly, we do not consider effective motion for a radial coordinate $r \in[0, \infty)$.
} 
Finally, our primary motivation came from phase-space quantum mechanics, or deformation quantization. In that context, we made some progress on solving the dynamical equations of Wigner functions, reported in [18. Here, we were able to demonstrate that in the sharp limit, our Wigner functions become those constructed by the Wigner transform from wave functions with Robin boundary conditions. Specifically, we showed that when $\alpha \rightarrow \infty$, equations (46, 47) reduce to the expected Wigner function, eqn. (44) [4], $x>0$. This justifies the assumption that the Wigner transform is unmodified for these examples of contact interactions.

\section{Acknowledgements}

This research was supported in part by a Discovery Grant from the Natural Sciences and Engineering Research Council of Canada and by the School of Graduate Studies of the University of Lethbridge.

M.W. thanks the Instituto de Matemáticas de UNAM in Morelia, México, for its warm hospitality. We also thank W. Chemissany, S. Das, A. Dasgupta and S. Sur for comments. 


\section{References}

[1] T. Fülöp, T. Cheon, I. Tsutsui, Phys. Rev. A 66 (2002) 052102 arXiv:quant-ph/0111057

[2] T. Cheon, T. Fülöp, I. Tsutsui, Ann. Phys. 294 (2001) 1 arXiv:quant-ph/0008123

[3] see, e.g.:

R. Blümel, Y. Dabaghian, R.V. Jensen, Phys. Rev. Lett. 88 (2002) 044101 arXiv:quant-ph/0107092;

Y. Dabaghian, R.V. Jensen, Eur. J. Phys. $26 \quad$ (2005) 423 arXiv:quant-ph/0407239];

P.L. Garrido, S. Goldstein, J. Lukkarinen, R. Tumulka, Paradoxical reflection in quantum mechanics, preprint (2008) arXiv:0808.0610]

[4] M.A. Walton, Ann. Phys. 322 (2007) 2233 arXiv:quant-ph/0609213

[5] G. Bonneau, J. Faraut, G. Valent, Am. J. Phys. 62 (2001) 322 arXiv:quant-ph/0103153v1];

V. S. Araujo, F. A. B. Coutinho, J. F. Perez, Am. J. Phys. 72 (2004) 203;

T. Fülöp, Singular potentials in quantum mechanics and ambiguity in the self-adjoint Hamiltonian, preprint (2007) arXiv:0708.0866

[6] A. Z. Capri, Am. J. Phys. (1977) 823

[7] F.A.B. Coutinho, Y. Nogami, J. Fernando Perez, J. Phys. A: Math. Gen. 32 (1999) L133; ibid 30 (1997) 3937

[8] S. Kryukov, M.A. Walton, Can. J. Phys. 84 (2006) 557 arXiv:quant-ph/0508005

[9] P. Šeba, Lett. Math. Phys. 10 (1985) 21 (see the example, page 23)

[10] H.E. Camblong, L.N. Epele, H. Fanchiotti, C.A. García Canal, C.R. Ordóñez, Phys. Lett. A364 (2007) 458 arXiv:hep-th/0604018

[11] C. Zachos, D. Fairlie, T. Curtright, Quantum Mechanics in Phase Space (World Scientific, 2005)

[12] J. Hancock, M.A. Walton, B. Wynder, Eur. J. Phys. 25 (2004) 525 arXiv:physics/0405029

[13] N.C. Dias, J.N. Prata, J. Math. Phys. 43 (2002) 4602 arXiv:quant-ph/0012140 
[14] S. Kryukov, M.A. Walton, Ann. Phys. 317 (2005) 474 arXiv:quant-ph/0412007

[15] N.C. Dias, J.N. Prata, Ann. Phys. $321 \quad$ (2006) 495 arXiv:quant-ph/0012140

[16] N.C. Dias, J.N. Prata, Deformation quantization of systems with boundaries, preprint (2006) arXiv:quant-ph/0612022;

N.C. Dias, A Posilicano, J.N. Prata, Self-adjoint, globally defined Hamiltonian operators for systems with boundaries, preprint (2007) arXiv:0707.0948,

[17] E. Cadena, R. Gilmore, Quantum mechanical reflection resonances, preprint (2006) arXiv:quant-ph/0610239

[18] B. Belchev, M.A. Walton, The Morse potential and phase-space quantum mechanics, preprint (2010) arXiv:1001.4816.

[19] A. Matsumoto, J. Phys. B: At. Mol. Opt. Phys. 21 (1988) 2863

[20] E.T. Whittaker, G.N. Watson, A Course of Modern Analysis, 4th ed. (Cambridge U. Press, 1962)

[21] A. P. Polyanin, V. F. Zaitsev, Handbook of Exact Solutions for Ordinary Differential Equations, 2nd ed. (Chapman \& Hall, 2003)

[22] M. Abramowitz and I. A. Stegun, Handbook of Mathematical Functions (National Bureau of Standards, 1972)

[23] A. Frank, A.L. Rivera, K.B. Wolf, Phys. Rev. A61 (2000) 054102(1-4) 